\title{
Increased cardiovascular risk of treated white coat and masked hypertension in patients with diabetes and chronic kidney disease: the HONEST Study
}

\author{
Toshio Kushiro ${ }^{1}$, Kazuomi Kario ${ }^{2}$, Ikuo Saito ${ }^{3}$, Satoshi Teramukai ${ }^{4}$, Yuki Sato ${ }^{5}$, Yasuyuki Okuda ${ }^{5}$ and \\ Kazuyuki Shimada ${ }^{6}$
}

The prognostic implications of treated white coat hypertension $(\mathrm{WCH})$ and masked hypertension $(\mathrm{MH})$ in patients with diabetes mellitus (DM) or chronic kidney disease (CKD) are not well documented. Using data from the HONEST study $(n=21591)$, we investigated the relationships between morning home systolic blood pressure (MHSBP) or clinic systolic blood pressure (CSBP) and cardiovascular (CV) risk in hypertensive patients with and without DM or CKD receiving olmesartan-based antihypertensive therapy. The study included 4426 DM patients and 4346 CKD patients at baseline who had 101 and 87 major CV events, respectively, during the follow-up. Compared with well-controlled non-DM patients (MHSBP $<135 \mathrm{~mm} \mathrm{Hg}$; CSBP $<140 \mathrm{~mm} \mathrm{Hg}$ ), DM patients with WCH (MHSBP $<135 \mathrm{~mm} \mathrm{Hg}$; CSBP $\geqslant 140 \mathrm{~mm} \mathrm{Hg}$ ), MH (MHSBP $\geqslant 135 \mathrm{~mm} \mathrm{Hg}$; CSBP $<140 \mathrm{~mm} \mathrm{Hg}$ ) or poorly controlled hypertension (PCH) (MHSBP $\geqslant 135 \mathrm{~mm} \mathrm{Hg}$; CSBP $\geqslant 140 \mathrm{~mm} \mathrm{Hg}$ ) had significantly higher CV risk (hazard ratio (HR), 2.73, 2.77 and 2.81, respectively). CV risk was also significantly increased in CKD patients with WCH, MH and PCH (HR, 2.14, 1.70 and 2.20, respectively) compared with well-controlled non-CKD patients. Furthermore, DM patients had significantly higher incidence rate than non-DM patients of MHSBP $\geqslant 125$ to $<135 \mathrm{~mm} \mathrm{Hg}(\mathrm{HR}, 1.98)$ and $\geqslant 135$ to $<145 \mathrm{~mm} \mathrm{Hg}$ (HR, 2.41). In conclusion, both WCH and MH are associated with increased CV risk, and thus control of both MHSBP and CSBP is important to reduce CV risk in DM or CKD patients. The results also suggest that even lower MHSBP $(<125 \mathrm{~mm} \mathrm{Hg}$ ) may be beneficial for DM patients, although this conclusion is limited by the small number of patients. Hypertension Research (2017) 40, 87-95; doi:10.1038/hr.2016.87; published online 11 August 2016

Keywords: cardiovascular diseases; chronic kidney disease; diabetes mellitus; masked hypertension; white coat hypertension

\section{INTRODUCTION}

Home blood pressure (BP) measurement and ambulatory BP monitoring are widely used in the diagnosis and treatment of hypertension. White coat hypertension (WCH) and masked hypertension $(\mathrm{MH})$ are diagnosed when there is a discrepancy between clinic BP (CBP) and home BP (HBP), ${ }^{1-3}$ and ambulatory $\mathrm{BP}$ monitoring is useful in diagnosing these types of hypertension. ${ }^{4}$

The concepts of $\mathrm{WCH}$ and $\mathrm{MH}$ were originally used to describe untreated hypertensive patients based on epidemiological findings to optimize antihypertensive treatment in these patients. For example, British and Japanese guidelines recommend nonpharmacological treatment for patients with $\mathrm{WCH}$ and pharmacological therapy for patients with $\mathrm{MH}^{5,6}$ For $\mathrm{WCH}$ patients who also have a metabolic abnormality or organ disorder, the European Society of Hypertension-European Society of Cardiology guidelines recommend pharmacological therapy. ${ }^{7}$ However, differences between BP measurements obtained at home or through ambulatory BP monitoring and CBP may persist despite receiving antihypertensive treatment. In such cases, the patients are described as having 'treated WCH' or 'treated MH'.

The Home BP measurement with Olmesartan Naive patients to Establish Standard Target blood pressure (HONEST) study is a large-scale, prospective, observational study involving more than 20000 Japanese patients with hypertension; the aim was to investigate the relationship between $\mathrm{HBP}$ and $\mathrm{CBP}$ and the incidence of cardiovascular $(\mathrm{CV})$ events in patients receiving olmesartan-based therapy. ${ }^{8}$ In our previous article describing the findings of the HONEST study, we reported a decrease in the proportion of patients with poorly controlled hypertension $(\mathrm{PCH})$, that is, patients whose HBP and CBP were both high, and a consequent increase in the proportion of patients with $\mathrm{WCH}$ or $\mathrm{MH}$ after treatment with olmesartan. ${ }^{9}$ After 16 weeks, the numbers of patients in both the $\mathrm{WCH}$ group and the $\mathrm{MH}$ group were approximately double the numbers at baseline. ${ }^{9,10}$

\footnotetext{
${ }^{1}$ The Life Planning Center Foundation, Tokyo, Japan; ${ }^{2}$ Division of Cardiovascular Medicine, Department of Medicine, Jichi Medical University School of Medicine, Shimotsuke, Japan; ${ }^{3}$ Keio University, Yokohama, Japan; ${ }^{4}$ Department of Biostatistics, Graduate School of Medical Science, Kyoto Prefectural University of Medicine, Kyoto, Japan; ${ }^{5}$ Daiichi Sankyo, Tokyo, Japan and ${ }^{6}$ Shin-Oyama City Hospital, Oyama, Japan

Correspondence: Professor T Kushiro, The Life Planning Center Foundation, 3-12-12, Mita, Minato-ku, Tokyo 108-0073, Japan.

E-mail: kushirot@gmail.com

Received 15 February 2016; revised 22 May 2016; accepted 31 May 2016; published online 11 August 2016
} 
Thus, we have shown that the prevalence of $\mathrm{WCH}$ and $\mathrm{MH}$ differs between untreated and treated patients. However, little information is available regarding $\mathrm{CV}$ risk in treated patients with $\mathrm{WCH}$ or $\mathrm{MH}$, and guidelines for the treatment of these patients remain unclear.

In patients with complications such as diabetes mellitus (DM) and chronic kidney disease (CKD), BP control is particularly important to prevent $\mathrm{CV}$ events. However, in most previous clinical studies, baseline CBP was used as an indicator of CV risk. Therefore, information is lacking on the relationship between on-treatment BP (especially HBP) and CV risk in patients with DM or CKD.

We previously reported the relationship between both morning HBP (MHBP) and CBP and CV risk during the follow-up period of the HONEST study. ${ }^{8}$ In the present analysis, we used MHBP and CBP data from the follow-up period to investigate these relationships in $\mathrm{WCH}$ and $\mathrm{MH}$ patients with and without DM or CKD.

\section{METHODS}

\section{Study design}

The HONEST study is a large-scale, prospective, observational study with a 2-year follow-up period. The study is registered at the University Hospital Medical Information Network (UMIN) Clinical Trials Registry (http://www.umin.ac.jp/ctr/index.htm) with the unique trial number UMIN000002567. The study protocol was approved by the Ethical Committee of Daiichi Sankyo, as well as the review boards of the participating institutions at their discretion. The study conforms to the pharmaceutical affairs laws of Japan and was approved by the Ministry of Health, Labour and Welfare of Japan. The study was carried out at registered medical institutions in compliance with Good Post-marketing Study Practice in Japan and the internal regulations for clinical studies at each institution. The objectives and methods of the HONEST study have been previously reported. ${ }^{11}$ Participants comprised olmesartan-naive outpatients with essential hypertension who had recorded their MHBP on $\geqslant 2$ days and CBP on $\geqslant 1$ day in the 28-day period before starting olmesartan therapy. All patients provided written informed consent. Patients had no specific target BP, and antihypertensive drugs, including olmesartan, were prescribed at the discretion of each patient's attending physician.

The present study was carried out by Daiichi Sankyo Co., Ltd (Tokyo, Japan) as part of the specific drug use results survey for olmesartan. Medical and statistical advisors provided advice on study protocol and study result interpretation. CV events were adjudicated by the Endpoint Committee, who were blinded to the HBP and CBP.

\section{Measurement of HBP}

In accordance with the 2009 Japanese Society of Hypertension Guidelines for the Management of Hypertension, ${ }^{12}$ patients used their own devices, according to the cuff oscillometric method, to measure their BP twice in the morning and twice at bedtime on 2 different days at each of the following measurement points: 1 week, 4 weeks, 16 weeks, 6 months, 12 months, 18 months, and 24 months. For the present analysis, we calculated the mean of the two daily MHBP measurements. Then, for each measurement point, we used the mean MHBP over 2 days.

To investigate relationships between HBP and incidence of CV events, on-treatment BP (mean BP during the follow-up period excluding baseline BP) was used. For patients who had had CV events, the mean BP measurements obtained until the first occurrence of such events was used.

\section{Measurement of CBP}

CBP was measured at 4 weeks, 16 weeks, 6 months, 12 months, 18 months, and 24 months. For each of these measurement points, one BP measurement was reported. The method used to measure CBP was at the discretion of the attending physicians.

Analyses of the relationship between CBP and the incidence of CV events were performed in the same way as for HBP.
Diagnosis of hypertension type based on patients' clinic and home blood pressure

In the present analysis, we defined the following four categories of hypertension status using clinic systolic BP (CSBP) and morning home systolic BP (MHSBP): $\mathrm{MH}$ was defined as CSBP $<140 \mathrm{~mm} \mathrm{Hg}$ and $\mathrm{MHSBP} \geqslant 135 \mathrm{~mm} \mathrm{Hg}$; WCH was defined as CSBP $\geqslant 140 \mathrm{~mm} \mathrm{Hg}$ and MHSBP $<135 \mathrm{~mm} \mathrm{Hg}$; PCH was defined as CSBP $\geqslant 140 \mathrm{~mm} \mathrm{Hg}$ and MHSBP $\geqslant 135 \mathrm{~mm} \mathrm{Hg}$; and well-controlled hypertension $(\mathrm{CH})$ was defined as CSBP $<140 \mathrm{~mm} \mathrm{Hg}$ and MHSBP $<135 \mathrm{~mm} \mathrm{Hg}$ (Figure 1). For each BP, the mean BP during the follow-up period excluding baseline BP was used.

\section{End points}

The primary end point for the present analysis was the first occurrence of a major CV event. This end point was a composite end point of stroke events (cerebral infarction, intracerebral hemorrhage, subarachnoid hemorrhage and unclassified stroke), cardiac events (myocardial infarction and coronary revascularization procedures for angina pectoris) and sudden death.

\section{Statistical analysis}

The statistical analysis population comprised eligible patients who received olmesartan at least once during the treatment period. Patients included in the present analysis were stratified according to the presence or absence of DM and CKD.

The presence of DM was determined by attending physicians who used clinical findings such as the use of hypoglycemic drugs and abnormal laboratory values. The presence of CKD was determined according to the following criteria: estimated glomerular filtration rate $<60 \mathrm{ml} \mathrm{min}^{-1}$ per

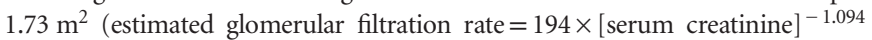
$\times$ age $^{-0.287} ; \times 0.739$ for female patients), qualitative proteinuria $\geqslant 2+$ or qualitative proteinuria $\geqslant 1+$ and renal disease.

For each comparison of baseline patient characteristics, categorical data were analyzed using the $\chi^{2}$ test, and quantitative data were analyzed using the $t$-test. For each subgroup, the incidence rate for $\mathrm{CV}$ events was estimated using the person-years method, and the results were compared by Poisson regression. The association between on-treatment BP and CV risk was analyzed using the multivariate Cox proportional hazards model, including BP, DM, CKD, interaction terms between $\mathrm{BP}$ and $\mathrm{DM}$ or CKD, sex, age, family history of $\mathrm{CV}$ disease, dyslipidemia, history of $\mathrm{CV}$ disease and smoking status as a covariate. The association between hypertension type based on on-treatment BP and $\mathrm{CV}$ risk was also analyzed using a multivariate Cox proportional hazards model.

All statistical tests were two sided using a significance level of 0.05 . SAS version 9.2 software (SAS Institute, Cary, NC, USA) was used for all statistical analyses.

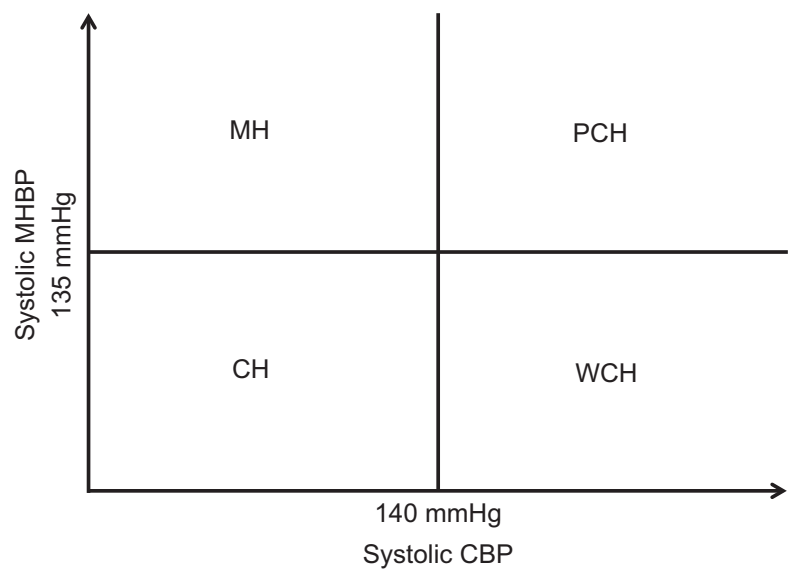

Figure 1 Categories of hypertension status used in the present study. $\mathrm{CBP}$, clinic blood pressure; $\mathrm{CH}$, well-controlled hypertension; $\mathrm{MH}$, masked hypertension; MHBP, morning home blood pressure; $\mathrm{PCH}$, poorly controlled hypertension; $\mathrm{WCH}$, white coat hypertension. 
Table 1A Comparison of baseline characteristics of patients with and without diabetes mellitus (DM)

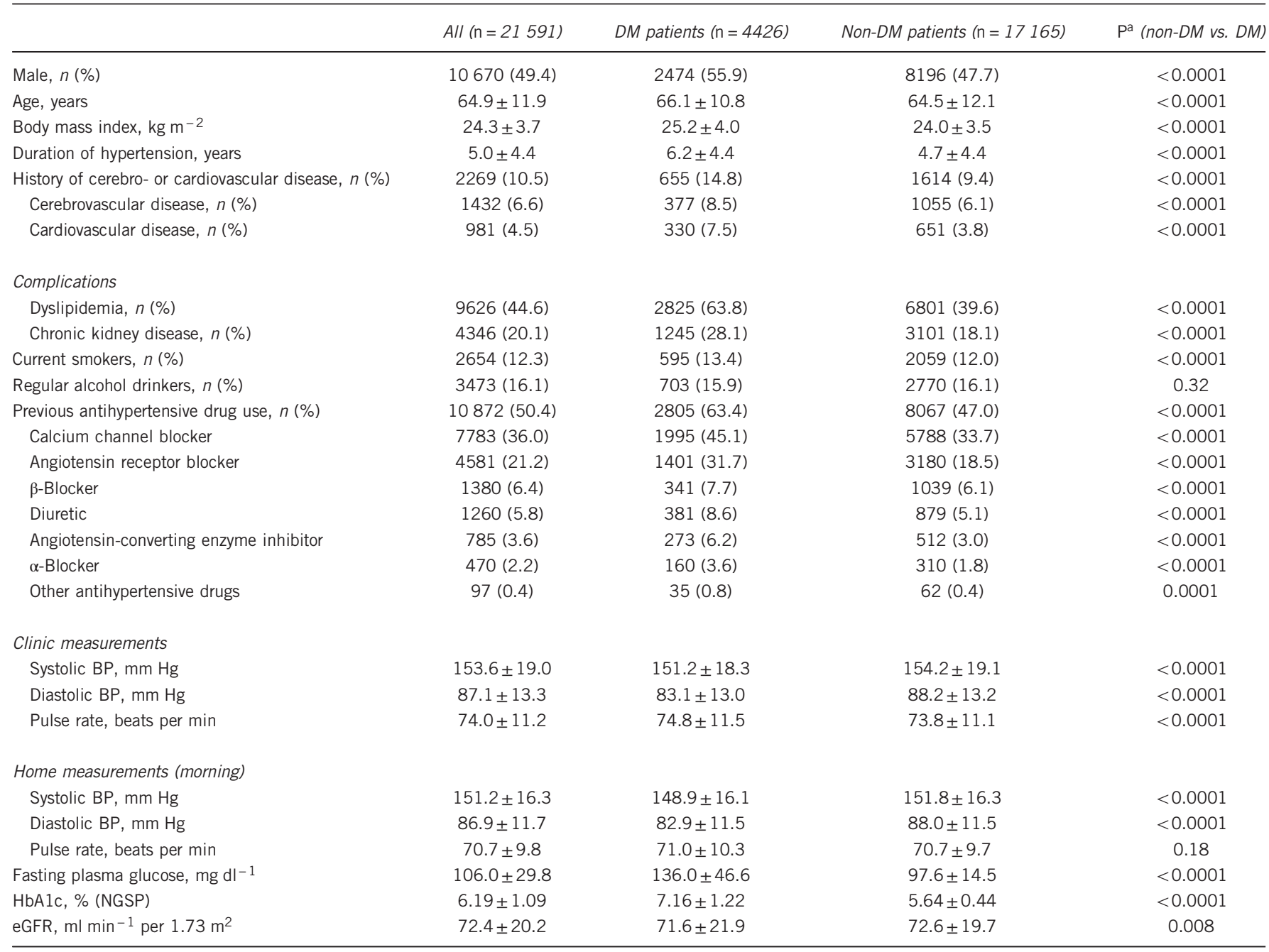

Abbreviations: BP, blood pressure; eGFR, estimated glomerular filtration rate; HbAlc, glycated hemoglobin Alc; NGSP, National Glycohemoglobin Standardization Program.

${ }^{a}$ Categorical data analyzed by $\chi^{2}$ test and quantitative data by unpaired $t$-test.

\section{RESULTS}

\section{Patient characteristics and changes in $\mathrm{BP}$}

Table 1A shows the baseline characteristics of all patients (the full analysis set, $n=21591$ ) whose data were used in the analysis comparing patients with and without DM. The group of DM patients contained a greater proportion of males, and the DM patients were older than the non-DM patients. The patients with DM had had hypertension longer than those without DM and were more likely to have previously received antihypertensive treatment. Baseline systolic and diastolic BP (both MHBP and CBP) were lower in DM patients than in non-DM patients by $\sim 3$ and $5 \mathrm{~mm} \mathrm{Hg}$, respectively. DM patients were more likely to have had a history of CV disease as well as concomitant dyslipidemia and CKD.

Table 1B shows the baseline characteristics of the 21428 patients whose data were included in the analysis comparing patients with and without CKD. Data from 163 patients in the full analysis set were excluded because their CKD status could not be determined. The groups of CKD and non-CKD patients had similar proportions of males and females, but CKD patients were $\sim 7$ years older than non-CKD patients. The patients with CKD had hypertension longer than those without CKD and were more likely to have previously received antihypertensive treatment. Baseline systolic and diastolic $\mathrm{BP}$ (both MHBP and CBP) were lower in CKD patients than in non-CKD patients by $\sim 2-3 \mathrm{~mm} \mathrm{Hg}$ and $4-5 \mathrm{~mm} \mathrm{Hg}$, respectively. $\mathrm{CKD}$ patients were more likely to have had a history of $\mathrm{CV}$ disease as well as concomitant dyslipidemia and DM.

Figure $2 \mathrm{a}$ and $\mathrm{b}$ shows changes in $\mathrm{BP}$ in DM and non-DM patients. Compared with non-DM patients, DM patients had significantly lower MHBP $(148.9 \pm 16.1 / 82.9 \pm 11.5 \mathrm{~mm} \mathrm{Hg} \quad v s$. $151.8 \pm 16.3 / 88.0 \pm 11.5 \mathrm{~mm} \mathrm{Hg})$ and CBP $(151.2 \pm 18.3 / 83.1 \pm$ $13.0 \mathrm{~mm} \mathrm{Hg}$ vs. $154.2 \pm 19.1 / 88.2 \pm 13.2 \mathrm{~mm} \mathrm{Hg}$ ) at baseline. BP in both DM and non-DM patients had reduced significantly by 16 weeks, and this reduction was maintained at 24 months.

Figure $2 \mathrm{c}$ and $\mathrm{d}$ shows changes in $\mathrm{BP}$ in $\mathrm{CKD}$ and non-CKD patients. Compared with non-CKD patients, $\mathrm{CKD}$ patients had significantly lower MHBP (149.7 \pm $17.2 / 83.4 \pm 11.8 \mathrm{~mm} \mathrm{Hg}$ vs. $151.6 \pm 16.0 / 87.9 \pm 11.4 \mathrm{~mm} \mathrm{Hg}) \quad$ and CBP $\quad(151.4 \pm 19.8 / 83.4 \pm 13.5 \mathrm{~mm} \mathrm{Hg} \quad$ vs. $\quad 154.2 \pm 18.7 / 88.1 \pm$ $13.1 \mathrm{~mm} \mathrm{Hg}$ ) at baseline. BP in both CKD and non-CKD patients had reduced significantly by 16 weeks, and this reduction was maintained at 24 months. 
Table 1B Comparison of baseline characteristics of patients with and without chronic kidney disease (CKD)

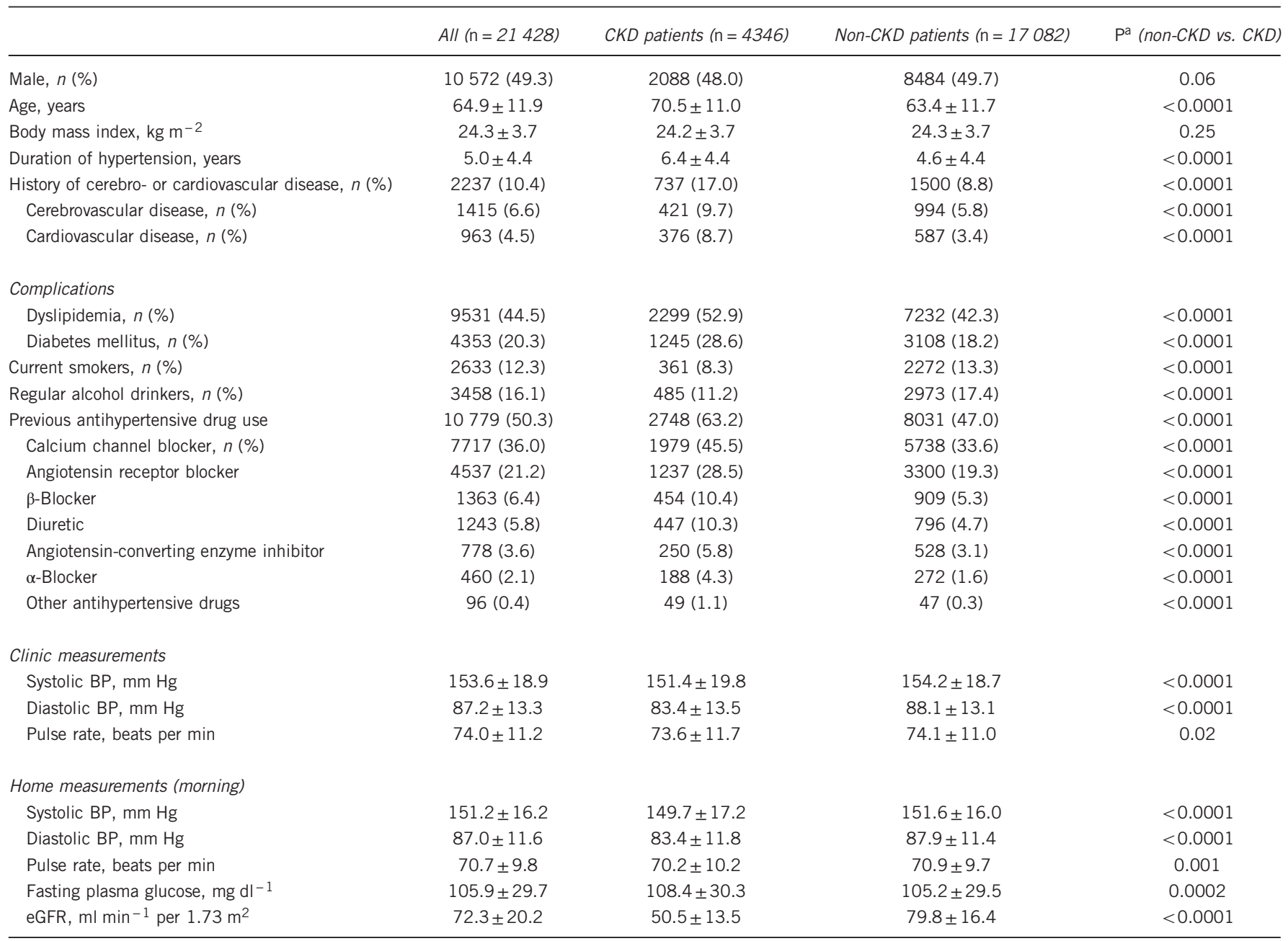

Abbreviations: $\mathrm{BP}$, blood pressure; eGFR, estimated glomerular filtration rate.

${ }^{a}$ Categorical data analyzed by $\chi^{2}$ test and quantitative data by unpaired $t$-test.

\section{$\mathrm{CV}$ risk in patients with and without diabetes}

Table 2A shows the incidence rate for CV events in DM and non-DM patients. CV events were more common in patients with DM than in those without DM (11.34/1000 vs. 5.20/1000 person-years, $P<0.0001)$. Of the major $\mathrm{CV}$ events, the incidence rate for cardiac events was particularly high in DM patients compared with that of non-DM patients $(6.26 / 1000$ vs. $1.88 / 1000$ person-years, $P<0.0001)$.

Figure $3 \mathrm{a}$ shows the relationship between the primary end point and MHSBP in DM and non-DM patients. When the patients with MHSBP $<125 \mathrm{mmHg}$ for non-DM patients were defined as a reference, the incidence rate was significantly higher in DM patients with MHSBP $\geqslant 135$ to $<145 \mathrm{~mm} \mathrm{Hg}$ and $\geqslant 155 \mathrm{~mm} \mathrm{Hg}$ and in nonDM patients with MHSBP $\geqslant 145$ to $<155 \mathrm{~mm} \mathrm{Hg}$ and $\geqslant 155 \mathrm{~mm} \mathrm{Hg}$ (hazard ratio (HR), 1.83-5.22). When we compared the incidence of $\mathrm{CV}$ events in DM patients with that in non-DM patients with the same achieved BP range, patients with DM had significantly higher incidence rate than non-DM patients at MHSBP $\geqslant 125$ to $<135 \mathrm{~mm} \mathrm{Hg}(\mathrm{HR}, 1.98,1.18-3.31, P=0.0092)$ and $\geqslant 135$ to $<145 \mathrm{~mm} \mathrm{Hg} \quad(\mathrm{HR}, 2.41,1.53-3.82, P=0.0002)$. There was a statistically significant interaction in the association of MHSBP with the primary end point between patients with and without DM (interaction $P=0.0376$ ). Figure $3 \mathrm{~b}$ shows the relationship between the primary end point and CSBP in DM and non-DM patients. When the patients with CSBP $<130 \mathrm{~mm} \mathrm{Hg}$ for non-DM patients were defined as a reference, the incidence rate was significantly higher in DM patients with CSBP $<130 \mathrm{~mm} \mathrm{Hg}, \geqslant 140$ to $<150 \mathrm{~mm} \mathrm{Hg}$, $\geqslant 150$ to $<160 \mathrm{~mm} \mathrm{Hg}$ and $\geqslant 160 \mathrm{~mm} \mathrm{Hg}$, as well as in non-DM patients with CSBP $\geqslant 150$ to $<160 \mathrm{~mm} \mathrm{Hg}$ and $\geqslant 160 \mathrm{~mm} \mathrm{Hg}$ (HR, 1.85-5.84). When we compared the incidence of CV events in DM patients with that of non-DM patients with the same BP range, patients with DM had a significantly higher incidence rate than non-DM patients at CSBP $<130 \mathrm{~mm} \mathrm{Hg}(\mathrm{HR}, 1.91)$ and $\geqslant 130$ to $<140 \mathrm{~mm} \mathrm{Hg}$ (HR, 1.70). No interaction between CSBP and the primary end point was found between patients with and without DM (interaction $P=0.5824$ ).

When the value for non-DM patients with $\mathrm{CH}$ was defined as a reference, the incidence rate was statistically significantly higher in DM patients with $\mathrm{WCH}, \mathrm{MH}$ and $\mathrm{PCH}(2.73,2.77$ and 2.81, respectively). In contrast, the incidence rate was statistically significantly higher in only non-DM patients with PCH (HR, 2.23; Figure 4a).

\section{$\mathrm{CV}$ risk in patients with and without $\mathrm{CKD}$}

Table 2B shows the incidence rate for CV events in $\mathrm{CKD}$ and non-CKD patients. CKD patients had a significantly higher incidence 
a
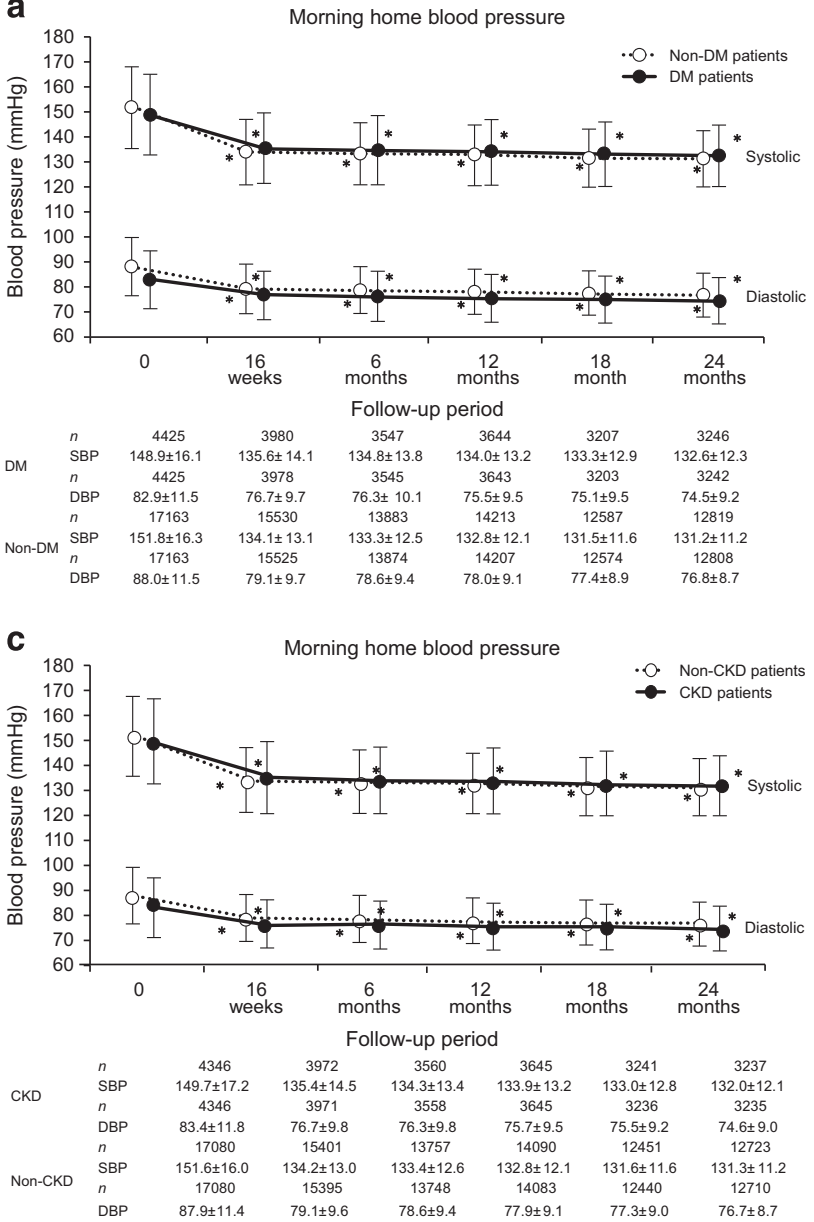

b

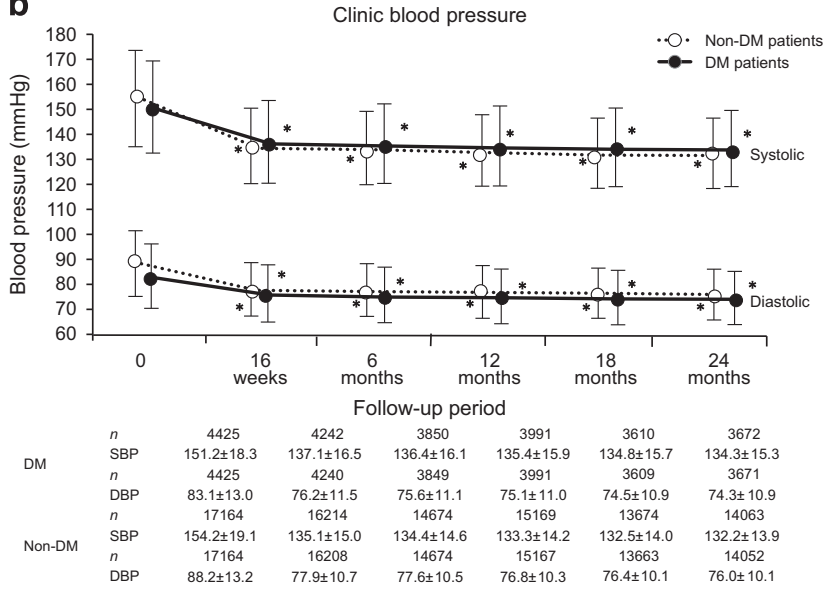

d

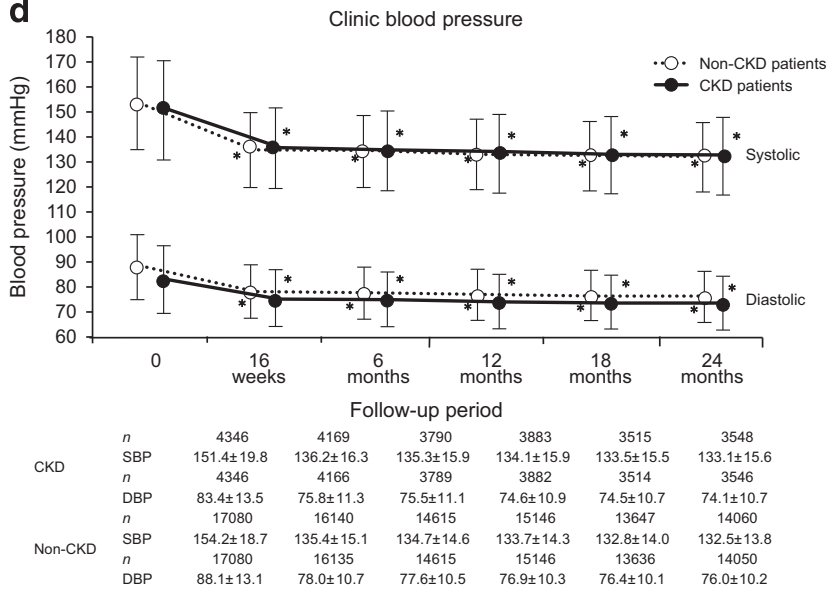

Figure 2 Changes in morning home and clinic blood pressure in patients with and without DM (a, b) and in patients with and without $C K D$ (c, d). ${ }^{*} P<0.001$ (vs. baseline; Dunnett - Hsu test). CKD, chronic kidney disease; DBP, diastolic blood pressure; DM, diabetes mellitus; SBP, systolic blood pressure.

rate for CV events than non-CKD patients $(10.06 / 1000$ vs. $5.44 / 1000$ person-years, $P<0.0001)$. Of the major $\mathrm{CV}$ events, both cerebrovascular and cardiac events were more common in patients with $\mathrm{CKD}$ than in those without $\operatorname{CKD}(P=0.0091$ and $P=0.0027$, respectively).

Figure $3 c$ shows the relationship between the primary end point and MHSBP in CKD and non-CKD patients. When the value of MHSBP $<125 \mathrm{~mm} \mathrm{Hg}$ for non-CKD patients was defined as a reference, the incidence rate was significantly higher in CKD patients with MHSBP $\geqslant 145$ to $<155 \mathrm{~mm} \mathrm{Hg}$ and $\geqslant 155 \mathrm{~mm} \mathrm{Hg}$, as well as in non-CKD patients with MHSBP $\geqslant 155 \mathrm{~mm} \mathrm{Hg}$ (HR, 2.18 - 5.95). Thus, CV risk tended to increase as MHSBP increased. Figure 3d shows the relationship between the primary end point and CSBP in CKD and non-CKD patients. Similarly, when the value of CSBP $<130 \mathrm{~mm} \mathrm{Hg}$ for non-CKD patients was defined as a reference, the incidence rate was significantly higher in CKD patients with CSBP $>160 \mathrm{~mm} \mathrm{Hg}$ and in non-CKD patients with CSBP $\geqslant 150$ to $<160 \mathrm{~mm} \mathrm{Hg}$ and $\geqslant 160 \mathrm{~mm} \mathrm{Hg}$ (HR, $1.83-6.62$ ). Thus, CV risk tended to increase as CSBP increased. When we compared the incidence of $\mathrm{CV}$ events in $\mathrm{CKD}$ patients with that of non-CKD patients with the same BP range (MHSBP or CSBP), no statistically significant difference in incidence rate was found between CKD and non-CKD patients across any subgroups (HR, $0.64-1.55)$. In the analysis of patients with and without $\mathrm{CKD}$, no interaction between either MHSBP or CSBP and the primary end point was found (interaction $P=0.5168$ and 0.8651 , respectively), suggesting that the association of MHSBP and CSBP with CV risk is similar in patients with and without $\mathrm{CKD}$. In a subsequent analysis, patients were classified into four groups according to hypertension type, and when the value for non$\mathrm{CKD}$ patients with $\mathrm{CH}$ was defined as a reference, $\mathrm{HR}$ was significantly higher in $\mathrm{CKD}$ patients with $\mathrm{WCH}, \mathrm{MH}$ and $\mathrm{PCH}(2.14,1.70$ and 2.20, respectively). In contrast, the incidence rate was statistically significantly higher only in non-CKD patients with PCH (HR, 2.19) (Figure 4b).

In CKD patients with concomitant DM $(n=1245)$ and those with concomitant proteinuria $(n=1238)$, the incidence rates for CV events were $17.50 / 1000$ and $15.87 / 1000$ person-years, respectively. When the value of patients with $\mathrm{CKD}$ alone and MHSBP $<125 \mathrm{~mm} \mathrm{Hg}$ was used as a reference, the HRs in patients with CKD+DM were 1.168 $(P=0.8583)$ at $<125 \mathrm{~mm} \mathrm{Hg}, 2.400(P=0.1366)$ at $\geqslant 125$ to $<135 \mathrm{~mm} \mathrm{Hg}, 2.886(P=0.0664)$ at $\geqslant 135$ to $<145 \mathrm{~mm} \mathrm{Hg}, 3.123$ $(P=0.0711)$ at $\geqslant 145$ to $<155 \mathrm{~mm} \mathrm{Hg}$ and $3.279(P=0.0958)$ at $\geqslant 155 \mathrm{~mm} \mathrm{Hg}$. With the same reference (MHSBP $<125 \mathrm{~mm} \mathrm{Hg}$ in patients with $\mathrm{CKD}$ alone), the HRs in patients with $\mathrm{CKD}+$ proteinuria were $2.661(P=0.2903)$ at $<125 \mathrm{~mm} \mathrm{Hg}, 2.494(P=0.2455)$ at $\geqslant 125$ to $<135 \mathrm{~mm} \mathrm{Hg}, 2.252(P=0.3017)$ at $\geqslant 135$ to $<145 \mathrm{~mm} \mathrm{Hg}, 3.135$ $(P=0.1656)$ at $\geqslant 145$ to $<155 \mathrm{~mm} \mathrm{Hg}$ and $4.742(P=0.0664)$ at $\geqslant 155 \mathrm{~mm} \mathrm{Hg}$.

\section{DISCUSSION}

In this analysis of data from the large-scale, prospective, observational HONEST study, we investigated the relationship between on-treatment MHBP and CBP and CV risk in patients with and 
Table 2A Cardiovascular events during the follow-up period in patients with and without diabetes mellitus (DM)

\begin{tabular}{|c|c|c|c|c|c|}
\hline & \multicolumn{2}{|r|}{ DM patients } & \multicolumn{2}{|r|}{ Non-DM patients } & \multirow{2}{*}{$P^{a}($ non-DM vs $D M$, } \\
\hline & & Incidence rate, events/1000 & & Incidence rate, events/1000 & \\
\hline Major cardiovascular events & 101 & 11.34 & 179 & 5.20 & $<0.0001$ \\
\hline Stroke events & 36 & 4.02 & 91 & 2.64 & 0.03 \\
\hline Atherothrombotic cerebral infarction & 12 & 1.33 & 31 & 0.90 & 0.24 \\
\hline Lacunar infarction & 14 & 1.56 & 26 & 0.75 & 0.03 \\
\hline Unclassified cerebral infarction & 4 & 0.44 & 9 & 0.26 & 0.37 \\
\hline Cerebral hemorrhage & 2 & 0.22 & 15 & 0.43 & 0.37 \\
\hline Subarachnoid hemorrhage & 3 & 0.33 & 5 & 0.14 & 0.25 \\
\hline Unclassified stroke & 1 & 0.11 & 2 & 0.06 & 0.59 \\
\hline Cardiac events & 56 & 6.26 & 65 & 1.88 & $<0.0001$ \\
\hline
\end{tabular}

apoisson regression was used.

Table 2B Cardiovascular events during the follow-up period in patients with and without chronic kidney disease (CKD)

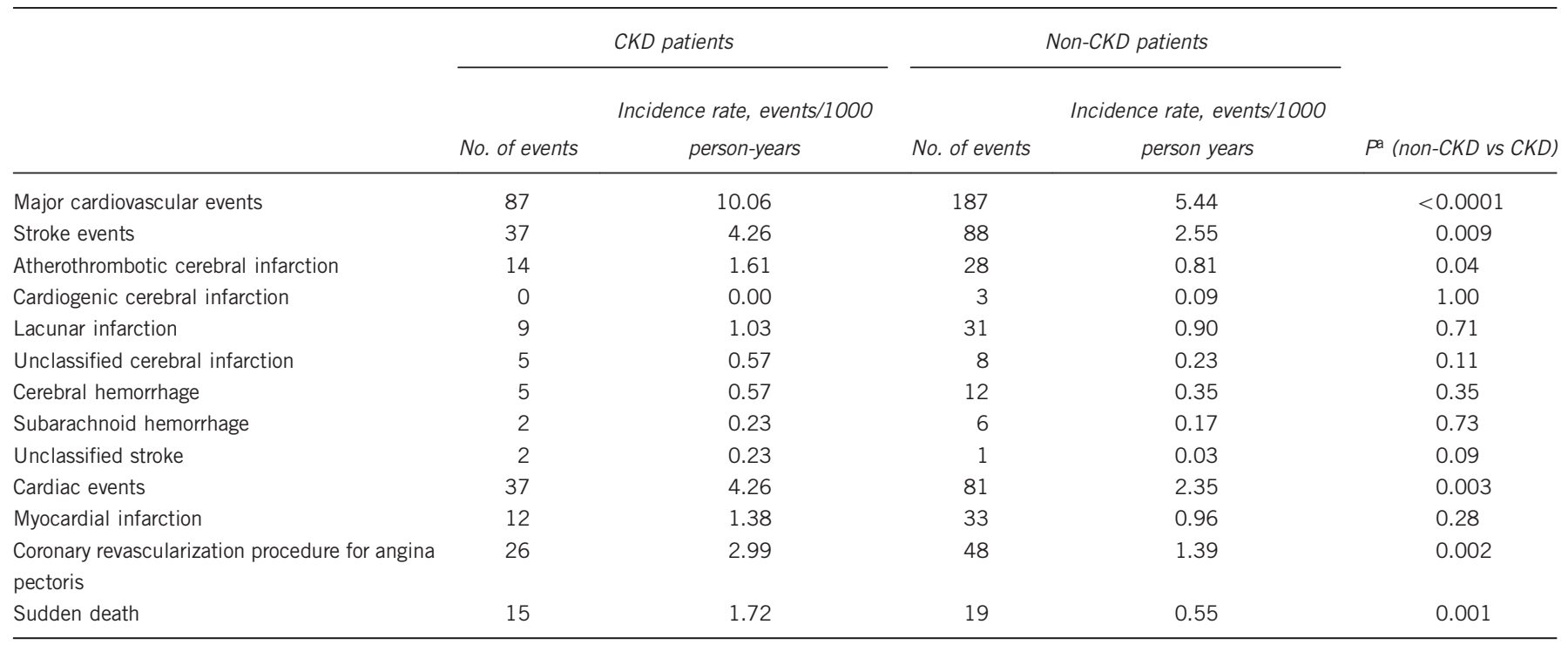

apoisson regression was used.

without DM or CKD in a real-world clinical setting. All patients had a similar reduction in BP during the follow-up period, regardless of concomitant DM or CKD. Both MHBP and CBP were independently related to $\mathrm{CV}$ risk. $\mathrm{WCH}$ and $\mathrm{MH}$ were also associated with increased $\mathrm{CV}$ risk when complicated with $\mathrm{DM}$ or $\mathrm{CKD}$. The findings indicate that control of both MHBP $(<135 \mathrm{~mm} \mathrm{Hg})$ and CBP $(<140 \mathrm{~mm} \mathrm{Hg})$ is important for reducing CV risk in patients with DM or CKD.

$\mathrm{CV}$ risk in patients with and without diabetes

Comparison of the baseline characteristics of patients with and without DM showed that DM patients were more likely to have $\mathrm{CV}$ risk factors such as older age, longer duration of hypertension, a history of CV disease and concomitant dyslipidemia or CKD.

The CV risk profile of patients with DM differed from that of those without DM. CV risk was higher in DM patients than in non-DM patients at MHSBP $\geqslant 125$ to $<145 \mathrm{~mm} \mathrm{Hg}$. However, at MHSBP $\geqslant 145 \mathrm{~mm} \mathrm{Hg}, \mathrm{CV}$ risk was similar in DM and non-DM patients. These findings suggest that $\mathrm{DM}$ is a greater risk factor for $\mathrm{CV}$ events than $\mathrm{BP}$ in the range of MHSBP $\geqslant 125$ to $<145 \mathrm{~mm} \mathrm{Hg}$. At MHSBP $\geqslant 145 \mathrm{~mm} \mathrm{Hg}$, however, BP makes a greater contribution to $\mathrm{CV}$ risk. In addition, although CV risk was significantly higher in DM patients than in non-DM patients at MHSBP $\geqslant 125$ to $<135 \mathrm{~mm} \mathrm{Hg}$, there was no difference between the two groups at $<125 \mathrm{~mm} \mathrm{Hg}$. Therefore, even lower MHSBP $(<125 \mathrm{~mm} \mathrm{Hg})$ may be beneficial for patients with DM, although the results were limited by the small number of patients with $<125 \mathrm{~mm} \mathrm{Hg}$ in the present study. The findings are consistent with those reported in previous studies; the HOMED-BP study $^{13}$ showed that MHPB $\geqslant 125 \mathrm{~mm} \mathrm{Hg}$ was significantly associated with increased CV risk in hypertensive patients with DM. Ushigome et al. ${ }^{14}$ reported that the optimal home systolic 

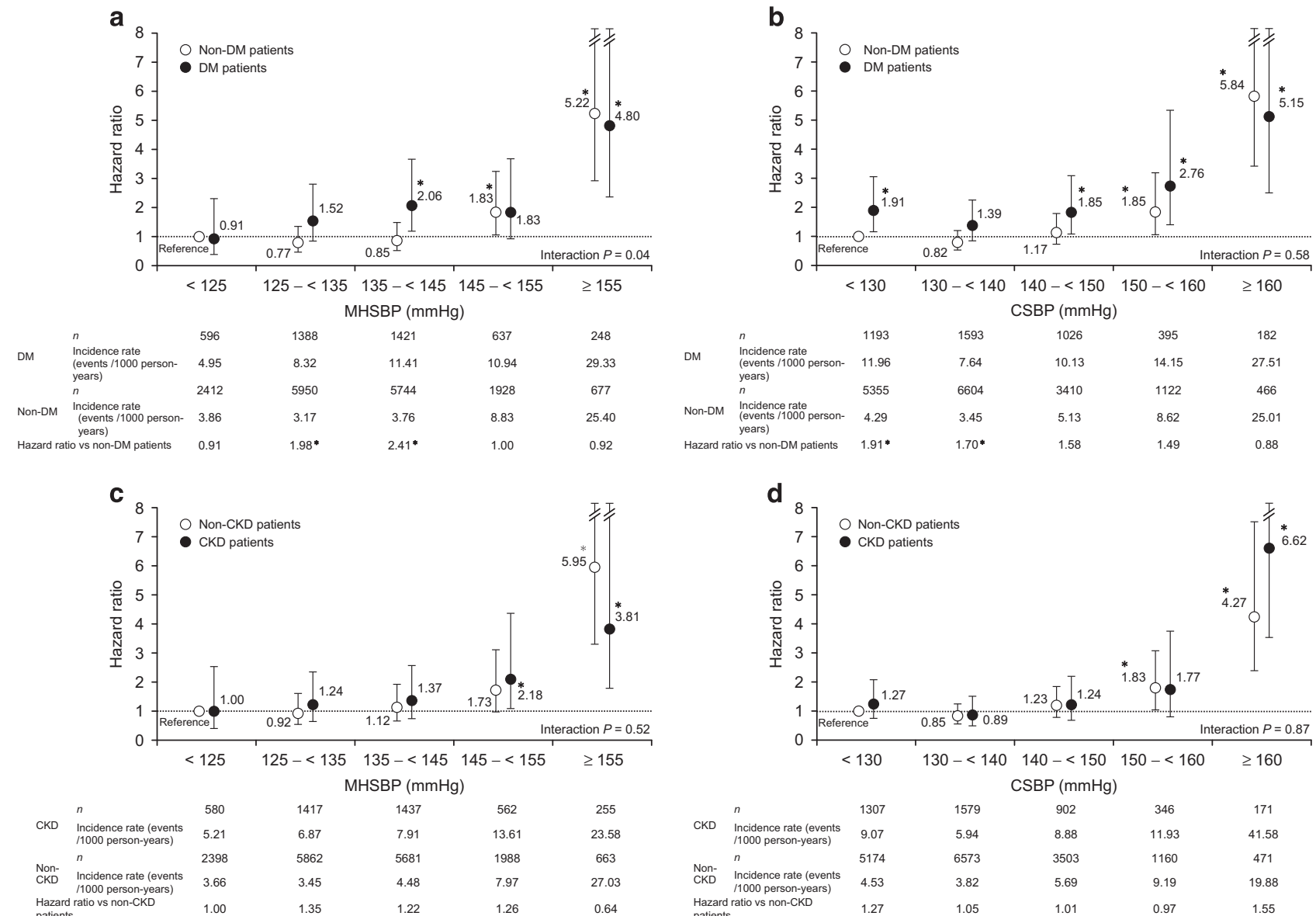

$\begin{array}{ll}307 & 1579 \\ 9.07 & 5.94 \\ 5174 & 6573 \\ 4.53 & 3.82 \\ 1.27 & 1.05\end{array}$

$\begin{array}{lccc}1579 & 902 & 346 & 171 \\ 5.94 & 8.88 & 11.93 & 41.58 \\ 6573 & 3503 & 1160 & 471 \\ 3.82 & 5.69 & 9.19 & 19.88 \\ 1.05 & 1.01 & 0.97 & 1.55\end{array}$

Figure 3 Relationship between the primary end point and morning home systolic blood pressure (a) or clinic systolic blood pressure (b) in patients with and without diabetes mellitus (DM; reference, $<125 \mathrm{~mm} \mathrm{Hg}$ (a) or $<130 \mathrm{~mm} \mathrm{Hg}$ (b) in non-DM patients; adjusted for sex, age, family history of cardiovascular disease, dyslipidemia, chronic kidney disease (CKD), history of cardiovascular disease and smoking status). Relationship between the primary end point and morning home systolic blood pressure (c) or clinic systolic blood pressure (d) in patients with and without CKD (reference, <125 mm Hg (c) or $<130 \mathrm{~mm} \mathrm{Hg}$ (d) in non-CKD patients; adjusted for sex, age, family history of cardiovascular disease, dyslipidemia, DM, history of cardiovascular disease and smoking status). ${ }^{*} P<0.05$. BP, blood pressure.
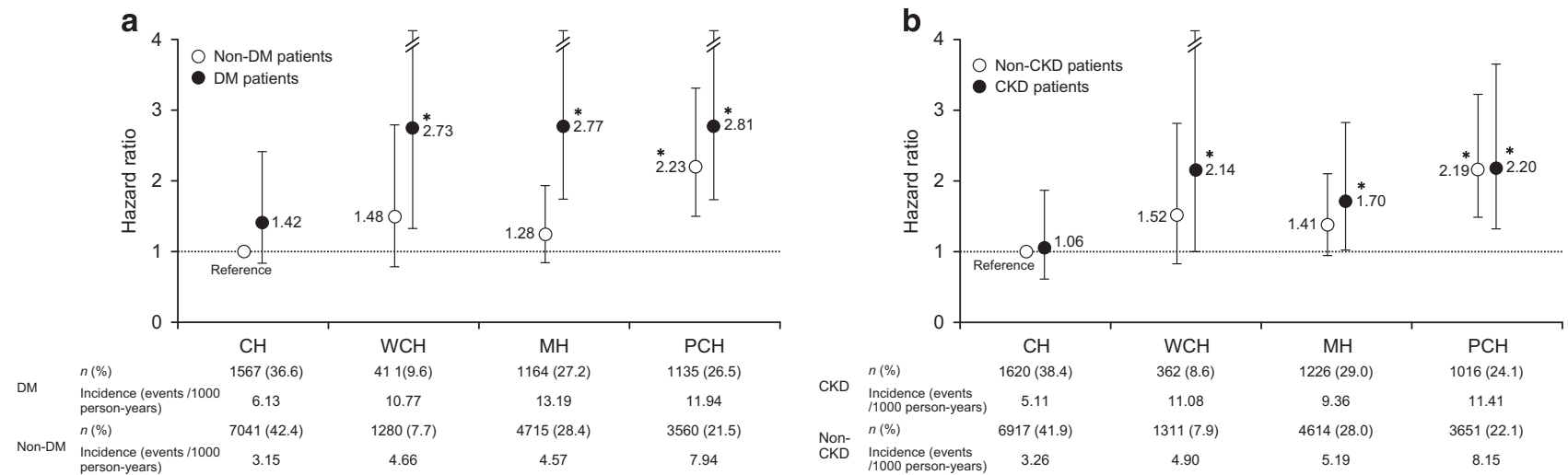

Figure 4 Primary end point in well-controlled hypertension $(\mathrm{CH})$, white coat hypertension (WCH), masked hypertension ( $\mathrm{MH}$ ) and poorly controlled hypertension (PCH) in patients with and without diabetes mellitus (DM) adjusted for sex, age, family history of cardiovascular disease, dyslipidemia, chronic kidney disease (CKD), history of cardiovascular disease and smoking status $\left({ }^{*} P<0.05\right.$ vs. non-DM patients with $\left.\mathrm{CH}\right)(\mathrm{a})$ and in patients with and without CKD adjusted for sex, age, family history of cardiovascular disease, dyslipidemia, DM, history of cardiovascular disease and smoking status $\left({ }^{\star} P<0.05\right.$ vs. non-CKD patients with $\mathrm{CH}$ (b). CBP, clinic blood pressure; MHBP, morning home blood pressure. 
BP target for prevention of progression of diabetic nephropathy in patients with DM was $<120 \mathrm{~mm} \mathrm{Hg}$.

In DM patients, CV risk was increased in the group of CSBP $<130 \mathrm{~mm} \mathrm{Hg}, \geqslant 140$ to $<150 \mathrm{~mm} \mathrm{Hg}, \geqslant 150$ to $<160 \mathrm{~mm} \mathrm{Hg}$ and $\geqslant 160 \mathrm{~mm} \mathrm{Hg}$. At CSBP $<140 \mathrm{~mm} \mathrm{Hg}, \mathrm{DM}$ contributes more than $\mathrm{BP}$ to $\mathrm{CV}$ risk. At CSBP $\geqslant 140 \mathrm{~mm} \mathrm{Hg}$, however, BP makes a larger contribution than DM.

$\mathrm{CV}$ risk was significantly higher for DM patients with $\mathrm{WCH}$ and $\mathrm{MH}$ than for non-DM patients with $\mathrm{CH}$. Therefore, patients with DM may have higher CV risk regardless of hypertension type, and control of both MHBP $(<135 \mathrm{~mm} \mathrm{Hg})$ and CBP $(<140 \mathrm{~mm} \mathrm{Hg})$ is particularly important in such patients. These results also suggest that even lower MHSBP $(<125 \mathrm{~mm} \mathrm{Hg})$ may be beneficial for DM patients, although this conclusion is limited by the small number of patients. In previous studies, the results of risk assessment in patients with $\mathrm{WCH}$ or $\mathrm{MH}$ have not been consistent, ${ }^{15-17}$ and this may be because of the difference in the numbers of hypertensive patients with concomitant DM included in these studies.

\section{$\mathrm{CV}$ risk in patients with and without $\mathrm{CKD}$}

The CV risk profile of patients with $\mathrm{CKD}$ was similar to that of patients without CKD. In both groups, CV risk increased for MHSBP $\geqslant 145$ to $<155 \mathrm{~mm} \mathrm{Hg}$ and for CSBP $\geqslant 150$ to $<160 \mathrm{~mm} \mathrm{Hg}$, with marked increases above these ranges.

In patients without $\mathrm{CKD}$, those with only $\mathrm{PCH}$ had significantly higher $\mathrm{CV}$ risk than that of non-CKD patients with $\mathrm{CH}$. In contrast, in patients with $\mathrm{CKD}$, those with $\mathrm{WCH}, \mathrm{MH}$ or $\mathrm{PCH}$ all had significantly higher $\mathrm{CV}$ risk than that of non-CKD patients with $\mathrm{CH}$.

To our knowledge, no study has investigated the relationship between $\mathrm{HBP}$ and $\mathrm{CV}$ risk in patients with CKD. Although it is difficult to determine the definitive target for MHSBP and CSBP in patients with concomitant CKD based on the results of the present study, our findings suggest that BP should be carefully monitored in patients with CKD when either MHSBP is $>135 \mathrm{~mm} \mathrm{Hg}$ or CSBP is $>140 \mathrm{~mm} \mathrm{Hg}$, as well as when both are increased.

The results of the present analysis show that the association of MHSBP and CSBP with CV risk is similar in patients with and without CKD. However, 2014 JSH (Japanese Society of Hypertension Guidelines for the Management of Hypertension) recommends a strict target CBP $(<130 / 80 \mathrm{~mm} \mathrm{Hg})$ for hypertensive patients with CKD with positive proteinuuria. ${ }^{6}$ Matsushita et al. ${ }^{18}$ also suggested that CV risk may be higher in hypertensive patients with CKD and proteinuria. Nevertheless, because the present study was an observational study under daily clinical practice and because laboratory tests were not mandatory, only 1238 patients $(5.7 \%$ of the full analysis set; $28.5 \%$ of CKD patients) were determined as having both CKD and proteinuria. Therefore, we report the results for patients with CKD regardless of the presence of proteinuria. In the HONEST study, the number of patients with both CKD and proteinuria was too small to analyze the relationship between on-treatment $\mathrm{BP}$ and CV risk. Nevertheless, in the present analysis, the event rate in patients with CKD and proteinuria was $15.87 / 1000$ person-years. Although no significant difference was found, the relationship between $\mathrm{CV}$ events and achieved BP was stronger compared with CKD patients without proteinuria. Further accumulation of patients would be necessary to determine the definitive target $\mathrm{BP}$ in patients with $\mathrm{CKD}$ and proteinuria.

Several studies have been carried out to investigate the effects of antihypertensive treatment on CV risk in hypertensive patients with DM and CKD. In the Irbesartan Diabetic Nephropathy Trial (IDNT) of DM patients, all-cause mortality in hypertensive patients with DM and CKD was found to be lowest at a systolic BP of $120-130 \mathrm{~mm}$ Hg. ${ }^{19}$ A meta-analysis of data from interventional studies of DM patients, including the Action to Control Cardiovascular Risk in Diabetes (ACCORD) study, showed that antihypertensive treatment prevents stroke in DM patients, of whom $40 \%$ had albuminuria. ${ }^{20}$ However, CBP was used as an indicator of CV risk in all these studies. The results of the present study suggested that monitoring and control of both CBP and MHBP may be useful in the management of CV risk in hypertensive patients with concomitant DM or CKD.

In conclusion, this analysis of the HONEST study indicates that both MHBP and CBP are independently related to CV risk regardless of white coat or masked hypertension types, and control of both MHBP $<135 \mathrm{~mm} \mathrm{Hg}$ and CBP $<140 \mathrm{~mm} \mathrm{Hg}$ is important to reduce $\mathrm{CV}$ risk in patients with $\mathrm{DM}$. In patients with $\mathrm{CKD}$, although a definitive target BP could not be determined in the present study, increased $\mathrm{CV}$ risk associated with $\mathrm{WCH}$ and $\mathrm{MH}$ was demonstrated. Thus, it is suggested that control of MHSBP $(<135 \mathrm{~mm} \mathrm{Hg})$ and CSBP $(<140 \mathrm{~mm} \mathrm{Hg})$ may also be important in these patients.

\section{Limitations}

The present analysis has several limitations. First, because the HONEST study is an observational study intended to reflect real-world clinical practice, patients were not blinded to treatment, and there was no control group. Further double-blind randomized controlled studies are necessary to determine specific BP targets for hypertensive patients with DM or CKD.

Second, because the HONEST study aimed to follow hypertensive patients in a daily clinical setting, details of laboratory tests were not prespecified, and the presence of proteinuria, as specified in JSH $2014,{ }^{6}$ was not mandatory. Therefore, there is a limitation in the evaluation of patients with $\mathrm{CKD}$ and/or proteinuria and the determination of a target BP in these patients. Third, the HONEST study included patients who already had cuff oscillometric HBP - measuring devices. The types of devices used and methods of HBP measurement were not specified. Nevertheless, HBP - measuring devices available in Japan have been validated and approved by the Ministry of Health, Labour and Welfare of Japan and are in accordance with the US (Association for the Advancement of Medical Instrumentation $)^{21}$ and European standards. ${ }^{22}$ In addition, HBP measurements were recorded by patients and reported to their physician, and the possibility of biased reporting by patients and physicians cannot be excluded completely. However, we consider their influence to be limited by the study design with its large sample size.

Finally, the mean follow-up period of the present study was short (2.02 years), and the CV risk profile may change over a longer study period. However, the HONEST study involved a large number of patients $(>20000)$, and the aggregated incidence rates for CV events per person-years are similar to or greater than those reported for previous studies.

\section{CONFLICT OF INTEREST}

Drs TK, KK, IS, ST and KS have received honoraria from Daiichi Sankyo, and Ms YS and Mr YO are employees of Daiichi Sankyo.

\section{ACKNOWLEDGEMENTS}

We gratefully acknowledge the numerous patients, investigators, fellows, nurses and research coordinators at each of the study sites who participated in the HONEST study. We also gratefully acknowledge their contributions to the study. This study was supported by funding from Daiichi Sankyo (Tokyo, Japan). Statistical analyses were carried out by the EPS Corporation (Tokyo, 
Japan) under the direction of the sponsor and the authors. Medical editorial assistance was provided by Macmillan Medical Communications, Tokyo, Japan, and was funded by Daiichi Sankyo.

Author contributions: The sponsor, Daiichi Sankyo, was involved in the designing, conducting, analyzing and reporting of the study. All authors contributed to writing or critically reviewing the manuscript, and all approved the final version for submission.

1 Liu JE, Roman MJ, Pini R, Schwartz JE, Pickering TG, Devereux RB. Cardiac and arterial target organ damage in adults with elevated ambulatory and normal office blood pressure. Ann Intern Med 1999; 131: 564-572.

2 Sega R, Trocino G, Lanzarotti A, Carugo S, Cesana G, Schiavina R, Valagussa F, Bombelli M, Giannattasio C, Zanchetti A, Mancia G. Alterations of cardiac structure in patients with isolated office, ambulatory, or home hypertension: data from the general population (Pressione Arteriose Monitorate E Loro Associazioni [PAMELA] Study). Circulation 2001; 104: 1385-1392.

3 Imai $Y$, Nagai K, Sakuma M, Sakuma H, Nakatsuka H, Satoh H, Minami N, Munakata M, Hashimoto J, Yamagishi T. Ambulatory blood pressure of adults in Ohasama, Japan. Hypertension 1993; 22: 900-912.

4 Gorostidi M, Vinyoles E, Banegas JR, de la Sierra A. Prevalence of white-coat and masked hypertension in national and international registries. Hypertens Res 2015; 38: 1-7.

5 National Institute of Health and Clinical Excellence. Hypertension: Clinical Management of Primary Hypertension in Adults. NICE Clinical Guideline 127. NICE: London, 2011. https://www.nice.org.uk/guidance/cg127. accessed 20 July 2016.

6 Shimamoto K, Ando K, Fujita T, Hasebe N, Higaki J, Horiuchi M, Imai Y, Imaizumi T, Ishimitsu $\mathrm{T}$, Ito $\mathrm{M}$, Ito $\mathrm{S}$, Itoh $\mathrm{H}$, Iwao $\mathrm{H}$, Kai $\mathrm{H}$, Kario $\mathrm{K}$, Kashihara $\mathrm{N}$, Kawano $\mathrm{Y}$, Kim-Mitsuyama S, Kimura G, Kohara K, Komuro I, Kumagai H, Matsuura H, Miura K, Morishita R, Naruse M, Node K, Ohya Y, Rakugi H, Saito I, Saitoh S, Shimada K, Shimosawa T, Suzuki H, Tamura K, Tanahashi N, Tsuchihashi T, Uchiyama M, Ueda S, Umemura S, Japanese Society of Hypertension Committee for Guidelines for the Management of Hypertension. The Japanese Society of Hypertension Guidelines for the Management of Hypertension (JSH 2014). Hypertens Res 2014; 37: 253-390.

7 Mancia G, Fagard R, Narkiewicz K, Redón J, Zanchetti A, Böhm M, Christiaens T, Cifkova R, De Backer G, Dominiczak A, Galderisi M, Grobbee DE, Jaarsma T, Kirchhof P, Kjeldsen SE, Laurent S, Manolis AJ, Nilsson PM, Ruilope LM, Schmieder RE, Sirnes PA, Sleight P, Viigimaa M, Waeber B, Zannad F, Task Force Members. 2013 ESH/ESC Guidelines for the Management of Arterial Hypertension: the Task Force for the Management of Arterial Hypertension of the European Society of Hypertension (ESH) and of the European Society of Cardiology (ESC). J Hypertens 2013; 31: 1281-1357.

8 Kario K, Saito I, Kushiro T, Teramukai S, Ishikawa Y, Mori Y, Kobayashi F, Shimada K. Home blood pressure and cardiovascular outcomes in patients during antihypertensive therapy: primary results of HONEST, a large-scale prospective, real-world observational study. Hypertension 2014; 64: 989-996.

9 Kario K, Saito I, Kushiro T, Teramukai S, Ishikawa Y, Hiramatsu K, Kobayashi F, Shimada K. Effect of the angiotensin II receptor antagonist olmesartan on morning home blood pressure in hypertension: HONEST study at 16 weeks. J Hum Hypertens 2013; 27: 721-728.

10 Kushiro T, Kario K, Saito I, Teramukai S, Mori Y, Okuda Y, Shimada K. Effectiveness of olmesartan-based treatment on home and clinic blood pressure in elderly patients with masked and white coat hypertension. Hypertens Res 2015; 38: $178-185$.

11 Saito I, Kario K, Kushiro T, Teramukai S, Zenimura N, Hiramatsu K, Kobayashi F, Shimada K. Rationale, study design, baseline characteristics and blood pressure at 16 weeks in the HONEST Study. Hypertens Res 2013; 36: 177-182.

12 Ogihara T, Kikuchi K, Matsuoka H, Fujita T, Higaki J, Horiuchi M, Imai Y, Imaizumi T, Ito $S$, Iwao $H$, Kario $K$, Kawano $Y$, Kim-Mitsuyama $S$, Kimura G, Matsubara $H$, Matsuura H, Naruse M, Saito I, Shimada K, Shimamoto K, Suzuki H, Takishita S, Tanahashi N, Tsuchihashi T, Uchiyama M, Ueda S, Ueshima H, Umemura S, Ishimitsu T, Rakugi HJapanese Society of Hypertension Committee. The Japanese
Society of Hypertension Guidelines for the Management of Hypertension (JSH 2009). Hypertens Res 2009; 32: 3-107.

13 Noguchi $Y$, Asayama K, Staessen JA, Inaba M, Ohkubo T, Hosaka M, Satoh M, Kamide K, Awata T, Katayama S, Imai Y, HOMED-BP study group. Predictive power of home blood pressure and clinic blood pressure in hypertensive patients with impaired glucose metabolism and diabetes. J Hypertens 2013; 31: 1593-1602.

14 Ushigome E, Hamaguchi M, Matsumoto S, Oyabu C, Omoto A, Tanaka T, Fukuda W, Hasegawa G, Shin-ichiMogami, Ohnishi M, Kitagawa Y, Tsunoda Sei, Oda Y, Nakamura N, Fukui M. Optimal home SBP targets for preventing the progression of diabetic nephropathy in patients with type 2 diabetes mellitus. J Hypertens 2015; 33. 1853-1859.

15 Bobrie G, Chatellier G, Genes N, Clerson P, Vaur L, Vaisse B, Menard J, Mallion JM. Cardiovascular prognosis of 'masked hypertension' detected by blood pressure self-measurement in elderly treated hypertensive patients. JAMA 2004; 291 . 1342-1349.

16 Hänninen MR, Niiranen TJ, Puukka PJ, Johansson J, Jula AM. Prognostic significance of masked and white-coat hypertension in the general population: the Finn-Home Study. J Hypertens 2012; 30: 705-712.

17 Stergiou GS, Asayama K, Thijs L, Kollias A, Niiranen TJ, Hozawa A, Boggia J, Johansson JK, Ohkubo T, Tsuji I, Jula AM, Imai Y, Staessen JA, International Database on HOme blood pressure in relation to Cardiovascular Outcome (IDHOCO) Investigators. Prognosis of white-coat and masked hypertension: International Database of HOme blood pressure in relation to Cardiovascular Outcome. Hypertension 2014; 63: 675-682.

18 Matsushita K, Coresh J, Sang Y, Chalmers J, Fox C, Guallar E, Jafar T, Jassal SK, Landman GW, Muntner P, Roderick P, Sairenchi T, Schöttker B, Shankar A, Shlipak M, Tonelli M, Townend J, van Zuilen A, Yamagishi K, Yamashita K, Gansevoort R, Sarnak M, Warnock DG, Woodward M, Ärnlöv J, CKD Prognosis Consortium. Estimated glomerular filtration rate and albuminuria for prediction of cardiovascular outcomes: a collaborative meta-analysis of individual participant data. Lancet Diabetes Endocrinol 2015; 3: 514-525.

19 Pohl MA, Blumenthal S, Cordonnier DJ, De Alvaro F, Deferrari G, Eisner G, Esmatjes E, Gilbert RE, Hunsicker LG, de Faria JB, Mangili R, Moore J Jr, Reisin E, Ritz E, Schernthaner G, Spitalewitz S, Tindall H, Rodby RA, Lewis EJ. Independent and additive impact of blood pressure control and angiotensin II receptor blockade on renal outcomes in the irbesartan diabetic nephropathy trial: clinical implications and limitations. J Am Soc Nephrol 2005; 16: 3027-3037.

20 Bangalore S, Kumar S, Lobach I, Messerli FH. Blood pressure targets in subjects with type 2 diabetes mellitus/impaired fasting glucose: observations from traditional and bayesian random-effects meta-analyses of randomized trials. Circulation 2011; 123 2799-2810.

21 Association for the Advancement of Medical Instrumentation. American National Standard. Electronic or Automated Sphygmomanometers. NSI/AAMI SP 10-1992. AAMI: Arlington, VA, 1993, 40.

22 European Committee for Standardization. Non-Invasive Sphygmomanometers. Part 3. Supplementary Requirements for Electromechanical Blood Pressure Measuring Systems. British Standard BS EN 1060-3: 1997. European Standard EN 1060-3 1997. ECS: Brussels, 1997

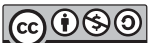

This work is licensed under a Creative Commons Attribution-NonCommercial-ShareAlike 4.0 International License. The images or other third party material in this article are included in the article's Creative Commons license, unless indicated otherwise in the credit line; if the material is not included under the Creative Commons license, users will need to obtain permission from the license holder to reproduce the material. To view a copy of this license, visit http://creativecommons.org/licenses/bync-sa/4.0/

(C) The Author(s) 2017 